\title{
MicroRNA-208a-3p participates in coronary heart disease by regulating the growth of hVSMCs by targeting BTG1
}

\author{
DONG WANG $^{1}$ and CAIYUN YAN ${ }^{2}$ \\ Departments of ${ }^{1}$ Cardiac Surgery and ${ }^{2}$ Nephrology, Shanxi Bethune Hospital, Taiyuan, Shanxi 030001, P.R. China
}

Received July 30, 2019; Accepted July 28, 2021

DOI: 10.3892/etm.2021.10994

\begin{abstract}
Human vascular smooth muscle cells (hVSMCs) are crucial in the progression of coronary heart disease (CHD). The present study aimed to investigate the role of microRNA-208a-3p (miR-208a-3p) in hVSMCs. Reverse transcription quantitative-PCR was performed to detect the levels of miR-208a-3p in the peripheral blood samples of patients with CHD and healthy volunteers. The results showed that miR-208a-3p was significantly upregulated in peripheral blood samples from patients with CHD compared with in healthy volunteers. Bioinformatics analysis and dual-luciferase reporter assays indicated that B-cell translocation gene 1 (BTG1) was a direct target gene of miR-208a-3p, and was downregulated in the peripheral blood samples of patients with CHD. Furthermore, this study also suggested that miR-208a-3p served an inhibitory role in the proliferation of hVSMCs, induced cell apoptosis, promoted the protein expression of Bax and reduced Bcl-2 protein expression; however, these effects were reversed by BTG1 silencing. In addition, the role of the PI3K/AKT pathway in mediating hVSMC apoptosis was examined via western blot analysis. Results indicated that inhibition of miR-208a-3p decreased phosphorylated (p)-AKT protein expression levels and the ratio of p-AKT/AKT in hVSMCs; however, BTG1-small interfering RNA abolished these effects. Taken together, these findings revealed that miR-208a-3p served a critical role in CHD development, regulating hVSMC function via targeting of BTG1, which was associated with the PI3K/AKT signaling pathway. Therefore, downregulated miR-208a-3p may serve as an ideal therapeutic target for CHD diagnosis and therapy.
\end{abstract}

\section{Introduction}

Cardiovascular disease, in particular coronary heart disease (CHD), has become the leading cause of death worldwide $(1,2)$.

Correspondence to: Dr Dong Wang, Department of Cardiac Surgery, Shanxi Bethune Hospital, 99 Longcheng Street, Taiyuan, Shanxi 030001, P.R. China

E-mail: wangdong1610@163.com

Key words: microRNA-208a-3p, vascular smooth muscle cells, coronary heart disease, B-cell translocation gene 1
Emerging evidence has demonstrated that dysfunction of human vascular smooth muscle cells (hVSMCs) serves an important role in the progression of angiocardiopathy, including CHD $(3,4)$. Numerous studies have indicated that abnormal regulation of VSMCs is crucial in the progression of CHD (3-5). Furthermore, a number of reports have indicated that microRNAs (miRNAs/miRs) are indispensable regulators of VSMC function $(6,7)$.

miRNAs, a family of small non-coding RNAs ( 22 nucleotides), can regulate gene expression by binding to the 3'-untranslated region (3'-UTR) of target mRNAs (8). miRNA functions have been reported in various biological behaviors, including cell proliferation, invasion and apoptosis (9). Previous reports have demonstrated that miRNAs are important regulators in the intricate biological progression of various cardiovascular diseases, including cardiac hypertrophy, heart failure, myocardial ischemia and reperfusion (10-14). However, to the best of our knowledge, little is known concerning the function of miR-208a-3p in the development of cardiovascular diseases in VSMCs. miR-208a-3p, the main sequence of miR-208a, has been reported to be associated with cardiovascular disease (15). Montgomery et al (16) have reported that downregulation of miR-208a improved cardiac function. In addition, miR-208 has been reported to be involved in various diseases, including colorectal cancer, gastric cancer and coronary artery disease $(15,17,18)$. However, the molecular mechanism of miR-208a-3p in CHD remains to be elucidated.

B-cell translocation gene 1 (BTG1) is member of the BTG anti-proliferative protein family, which may promote cytochrome $c$ translocation in mitochondria (19). In addition, it is a vital cofactor regulating certain biological behaviors, including cell proliferation and apoptosis (19). However, the potential role of BTG1 in VSMCs remains unclear.

The aim of the present study was to investigate the role of miR-208a-3p in CHD by examining its functional role in the regulation of hVSMC proliferation, and further examine the molecular mechanism. This study may provide a novel diagnostic and treatment target in CHD therapy.

\section{Materials and methods}

Clinical specimen collection. Peripheral blood samples ( $2 \mathrm{ml} /$ individual) were extracted from 30 patients with CHD (age range, 43-75 years; 20 males and 10 females) and 30 healthy donors (age range, 46-77 years; 20 males and 
10 females) at Shanxi Dayi Hospital between February 2017 and August 2018. The samples were immediately frozen and stored at $-80^{\circ} \mathrm{C}$ until further use. Written informed consent was provided from all participants approving the use of their samples in the present study. All study protocols were approved by the Ethics Committee of Shanxi Bethune Hospital (Taiyuan, China). The inclusion criteria were as follows: Coronary angiography suggested that at least one main coronary artery or main branch had obvious coronary artery stenosis $\geq 50 \%$, and the conclusion was judged by at least two experienced cardiologists. The exclusion criteria were as follows: i) Patients with myocardial bridge, cardiomyopathy, valvular heart disease or acute myocardial infarction; ii) patients with hypothyroidism, hyperthyroidism, hypothalamic or pituitary disease and other endocrine diseases; iii) patients with cancer, acute cerebrovascular disease, severe infection, liver or kidney dysfunction and hereditary hyperlipidemia; and iv) patients with history of mental illness or family history of mental illness.

Cell culture. hVSMCs were purchased from American Type Culture Collection. Cells were maintained in Dulbecco's modified Eagle's medium (HyClone; Cytiva) supplemented with $10 \%$ fetal bovine serum (FBS; Gibco; Thermo Fisher Scientific, Inc.), $100 \mathrm{U} / \mathrm{ml}$ penicillin and $100 \mathrm{mg} / \mathrm{ml}$ streptomycin at $37^{\circ} \mathrm{C}$ with $5 \% \mathrm{CO}_{2}$.

Cell transfection and reagents. A total of $100 \mathrm{nM}$ miR-208a-3p inhibitor (5'-ACA AGCUUUUUGCUCGUC UUAU-3'; Shanghai GenePharma Co., Ltd.), $100 \mathrm{nM}$ inhibitor control (5'-CAGUACUUUUGUGUAGUACAA-3'; Shanghai GenePharma Co., Ltd.), $1 \mu \mathrm{M}$ control-small interfering (si)RNA (cat. no. sc-36869; Santa Cruz Biotechnology, Inc.), $0.5 \mu \mathrm{M}$ BTG1-siRNA (cat. no. sc-43644; Santa Cruz Biotechnology, Inc.), $100 \mathrm{nM}$ miR-208a-3p inhibitor $+1 \mu \mathrm{M}$ control-siRNA or $100 \mathrm{nM}$ miR-208a-3p inhibitor $+0.5 \mu \mathrm{M}$ BTG1-siRNA was transfected in hVSMCs $\left(5 \times 10^{4}\right.$ cells/well $)$ using Lipofectamine ${ }^{\circledR} 2000$ Reagent (Invitrogen; Thermo Fisher Scientific, Inc.) for $48 \mathrm{~h}$, according to the manufacturer's protocols. The transfection efficiency was evaluated via reverse transcription-quantitative PCR (RT-qPCR).

MTT assay. An MTT assay was performed to assess the cell viability of hVSMCs. The hVSMCs $\left(6 \times 10^{3}\right.$ cells/well $)$ were cultured in 96-well plates (BD Biosciences) in triplicate for $24 \mathrm{~h}$ at $37^{\circ} \mathrm{C}$. DMEM was removed and miR-208a-3p inhibitor, inhibitor control, miR-208a-3p inhibitor + control-siRNA, or miR-208a-3p inhibitor + BTG1-siRNA in $100 \mu 1$ of fresh medium were added, and cells were subsequently cultured for $48 \mathrm{~h}$ at $37^{\circ} \mathrm{C}$. MTT solution $(10 \mu \mathrm{l})$ was added to each well and incubated for a further $4 \mathrm{~h}$. A total of $100 \mu \mathrm{l}$ DMSO was added to solubilize the formazan product. The optical density (OD) of hVSMCs was determined at a wavelength of $490 \mathrm{~nm}$ by a Synergy ${ }^{\mathrm{TM}} 2$ Multi-function microplate reader (Bio-Tek Instruments, Inc.).

Cell apoptosis assay. hVSMCs were transfected with miR-208a-3p inhibitor, inhibitor control, miR-208a-3p inhibitor + control-siRNA or miR-208a-3p inhibitor + BTG1-siRNA for $48 \mathrm{~h}$, and cells were subsequently harvested. Cell apoptosis analysis was conducted using Annexin V-FITC/propidium iodide dual staining at $4^{\circ} \mathrm{C}$ for $30 \mathrm{~min}$ in the dark, according to the manufacturer's protocols. Cell apoptosis (early apoptosis + late apoptosis) was analyzed using a FACS flow cytometer (BD Biosciences) and the results were analyzed using FlowJo ${ }^{\mathrm{TM}}$ v7.6.1 software (BD Biosciences).

Dual-luciferase reporter assay. Bioinformatics analysis using TargetScan (version 7.2; http://www.targetscan.org/vert_72/) was performed to predict the target gene of miR-208a-3p. The binding site between the 3'-UTR of BTG1 and miR-208a-3p was subsequently investigate. To validate the binding site, a dual-luciferase reporter assay was performed. DNA segments in the 3'-UTR of BTG1 containing the miR-208a-3p binding site were cloned into a pmirGLO dual-luciferase vector (Promega Corporation) to conduct the pmirGLO-BTG1 wild-type plasmid (BTG1-WT). The mutant (MUT) 3'-UTR of BTG1 was also cloned into pmirGLO to generate BTG1-MUT plasmids. $1 \mu \mathrm{g}$ BTG1-WT and $1 \mu \mathrm{g}$ BTG1-MUT were co-transfected into hVSMCs $\left(5 \times 10^{4}\right.$ cells) with $100 \mathrm{nM}$ miR-208a-3p mimic (sense, 5'-AUAAGACGAGCAAAAAGC UUGU-3'; and antisense, 5'-AGCUUUUUGCUCGUCUUA UUU-3'; Shanghai GenePharma Co., Ltd.) or $100 \mathrm{nM}$ mimic control (sense, 5'-UUCUCCGAACGUGUCACGUTT-3'; and antisense, 5'-ACGUGACACGUUCGGAGAATT-3'; Shanghai GenePharma Co., Ltd.) using Lipofectamine 2000 according to the manufacturer's protocols. Following $48 \mathrm{~h}$ of transfection, luciferase activity was detected using a Dual-Luciferase Reporter Assay System (Promega Corporation). Firefly luciferase activities were normalized to Renilla luciferase activity.

$R N A$ isolation and RT-qPCR. Total RNA from cultured cells or blood specimens was extracted using TRIzol ${ }^{\circledR}$ reagent (Invitrogen; Thermo Fisher Scientific, Inc.) according to the manufacturer's protocol. Total RNA (200 ng) was used to produce cDNA using PrimeScript RT Master Mix (Takara Biotechnology Co.,Ltd.) using the RT primer 5'-CTCAACTGG TGTCGTGGAGTCGGCAATTCAGTTGAGACAAGCTT-3'. A SYBR PrimeScript RT-PCR kit (Takara Biotechnology Co., Ltd.) was used to evaluate the miR-208a-3p and BTG1 expression levels. The relative expression of mRNA or miR-208a-3p was normalized to GAPDH or U6, respectively. The thermocycling conditions were as follows: Initial denaturation at $95^{\circ} \mathrm{C}$ for $5 \mathrm{~min}$, followed by 35 cycles of $94^{\circ} \mathrm{C}$ for $15 \mathrm{sec}$, $50^{\circ} \mathrm{C}$ for $30 \mathrm{sec}, 72^{\circ} \mathrm{C}$ for $30 \mathrm{sec}$; and final extension at $72^{\circ} \mathrm{C}$ for $10 \mathrm{~min}$. Primer sequences for PCR were listed as follows: miR-208a-3p forward, 5'-GGGCCATAAGACGAGCAAA-3' and miR-208a-3p stemloop reverse, 5'-CTCAACTGGTGT CGTGGAGTC-3'; U6 forward, 5'-TGCGGGTGCTCGCTT CGCAGC-3' and reverse, 5'-CCAGTGCAGGGTCCGAGG T-3'; GAPDH forward, 5'-CGGAGTCAACGGATTTGGTCG TAT-3' and reverse, 5'-AGCCTTCTCCAGGTGGTGAAG AC-3'; BTG1 forward, 5'-CATCTCCAAGTTTCTCCGCAC C-3' and reverse, 5'-GCGAATACAACGGTAACCCGATC-3'. The results were calculated using the $2^{-\Delta \Delta C q}$ method (20).

Western blot analysis. hVSMCs were harvested and lysed using RIPA lysis buffer (Beyotime Institute of Biotechnology). The concentration of total protein was calculated using a BCA Protein Assay kit (Thermo Fisher Scientific, Inc.). The protein 
fractions were subsequently mixed with $5 \mathrm{X}$ SDS, boiled and centrifuged at $10,000 \times \mathrm{g}$ at $4^{\circ} \mathrm{C}$ for $5 \mathrm{~min}$. A total of $40 \mu \mathrm{g}$ proteins per lane were subjected to $10 \%$ SDS-PAGE. The proteins were transferred onto PVDF membranes and were subsequently incubated with primary antibodies against BTG1 (cat. no. ab151740; Abcam), Bcl-2 (cat. no. ab185002; Abcam), Bax (cat. no. ab32503; Abcam), phosphorylated (p)-AKT (cat. no. 4060; Cell Signaling Technology, Inc.), AKT (cat. no. 4691; Cell Signaling Technology, Inc.) and GAPDH (cat. no. 5174; Cell Signaling Technology, Inc.) at a dilution of $1: 1,000$ at $4^{\circ} \mathrm{C}$ overnight. After washing with PBS- $0.1 \%$ Tween 20 , the membranes were incubated with HRP-conjugated goat anti-rabbit secondary antibody (1:2,000; cat. no. 7074; Cell Signaling Technology, Inc.) for $1 \mathrm{~h}$ at room temperature. The proteins bands were analyzed using Pierce ${ }^{\mathrm{TM}}$ ECL Western Blotting Substrate (Pierce; Thermo Fisher Scientific, Inc.), according to the manufacturer's protocol. The intensity of protein bands was quantified using Image $\mathrm{Lab}^{\mathrm{TM}}$ Software (version 5.2.1; Bio-Rad Laboratories, Inc.).

Statistical analysis. Each experiment was repeated in triplicate. Data were presented as the mean \pm standard deviation. Statistical analysis was carried out using SPSS software version 16.0 (SPSS, Inc.). The differences between two groups were assessed by Student's t-test, and comparisons between multiple groups were detected using one-way ANOVA followed by Tukey's post hoc test. $\mathrm{P}<0.05$ was considered to indicate a statistically significant difference.

\section{Results}

miR-208a-3p expression is upregulated in the peripheral blood of patients with CHD. In order to explore the clinical relevance of miR-208a-3p in CHD, the expression level of miR-208a-3p was first determined in the peripheral blood samples of 30 patients with CHD and 30 healthy volunteers via RT-qPCR analysis. The results showed that significantly higher miR-208a-3p expression was detected in the peripheral blood samples of patients with CHD compared with healthy donors, indicating that miR-208a-3p may participate in CHD development (Fig. 1).

miR-208a-3p directly targets to the 3'-UTR of BTG1. Bioinformatics analysis was used to identify potential miR-208a-3p target genes. It was found that miR-208-3p has hundreds of potential target genes, including BTG1, with a putative binding site within its 3'-UTR for miR-208a-3p (Fig. 2A). As abnormal growth of VSMCs is crucial in the progression of CHD (5), and BTG1 serves important roles in regulating cell proliferation (17). However, the potential mechanism of BTG1 in VSMCs remains unclear. Thus, BTG1 was selected for further investigation. A luciferase reporter assay was subsequently conducted to verify whether miR-208a-3p could directly target to BTG1. Results indicated that miR-208a-3p mimic significantly enhanced miR-208a-3p expression in hVSMCs compared with the mimic control (Fig. 2B). Furthermore, miR-208a-3p overexpression significantly inhibited the luciferase activity of BTG1-WT. However, the luciferase activity of BTG1-MUT was not notably affected by co-transfection with miR-208-3p mimic compared with

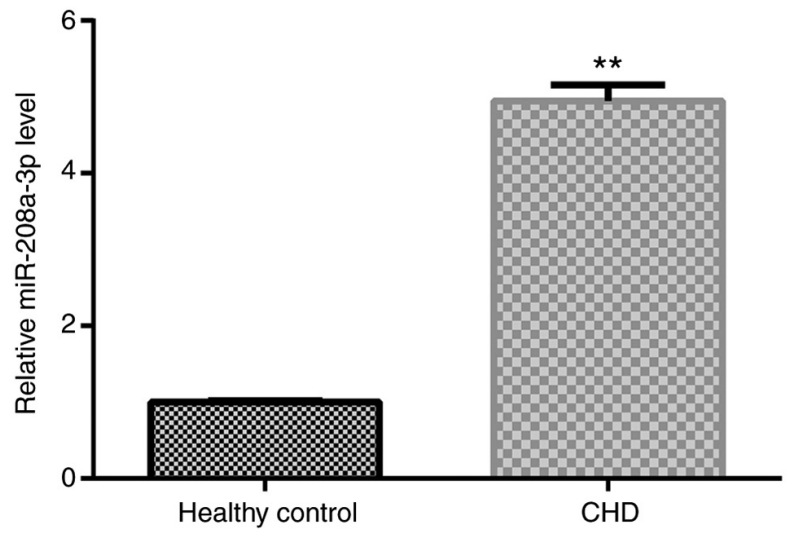

Figure 1. miR-208a-3p is upregulated in the peripheral blood samples of patients with $\mathrm{CHD}$. $\mathrm{N}=30$ /group. ${ }^{* *} \mathrm{P}<0.01$ vs. healthy control. $\mathrm{CHD}$, coronary heart disease; miR-208a-3p, microRNA-208a-3p.

mimic control, suggesting that miR-208a-3p directly targeted the 3'-UTR of BTG1 (Fig. 2C).

To further investigate BTG1 expression in patients with CHD, RT-qPCR analysis was conducted to evaluate the mRNA expression levels of BTG1 in the blood samples of patients with CHD. The findings suggested that the BTG1 mRNA level was significantly downregulated in patients with CHD compared with healthy individuals (Fig. 2D). Therefore, BTG1 was hypothesized to serve as a functional target of miR-208a-3p in CHD.

miR-208a-3p negatively regulates BTG1 expression in hVSMCs. The effect of miR-208a-3p on BTG1 expression was subsequently investigated in hVSMCs. Control-siRNA, BTG1-siRNA, miR-208a-3p inhibitor or inhibitor control were transfected into hVSMCs for $48 \mathrm{~h}$ and transfection efficiency was evaluated via RT-qPCR analysis. As shown in Fig. 3A, miR-208a-3p inhibitor significantly suppressed miR-208a-3p levels in hVSMCs compared with the control group. In addition, in hVSMCs, BTG1-siRNA significantly downregulated the mRNA expression of BTG1 compared with the control group (Fig. 3B). Further experiments demonstrated that miR-208a-3p inhibitor was able to upregulate BTG1 at both the mRNA and the protein level, and this upregulation was reversed by BTG1-siRNA (Fig. 3C-E). These findings suggested that miR-208a-3p negatively regulated BTG1 expression in hVSMCs.

BTG1-siRNA abolishes the effect of miR-208a-3p inhibitor on cell viability and apoptosis in hVSMCs. To further assess the role of miR-208a-3p in hVSMCs, MTT and flow cytometry assays were performed to determine hVSMC viability and apoptosis, respectively. miR-208a-3p inhibitor, inhibitor control, miR-208a-3p inhibitor + control-siRNA or miR-208a-3p inhibitor + BTG1-siRNA were transfected into hVSMCs. MTT assay results suggested that miR-208a-3p inhibitor significantly inhibited cell viability compared with the control group, while miR-208a-3p inhibitor and BTG1-siRNA co-transfection significantly promoted VSMC viability compared with the miR-208a-3p inhibitor group (Fig. 4A). Furthermore, flow cytometry analysis was carried out to evaluate the effect of miR-208a-3p inhibitor on hVSMC apoptosis. The results 


\section{A}

Position 1370-1376 of BTG1 3' UTR $5^{\prime}$, ... GGCAGGGGACCCAUAGLCLUAAA. .

hsa-miR-208a-3p 3' LGULCGAAAAAGGAGCAGAAUA
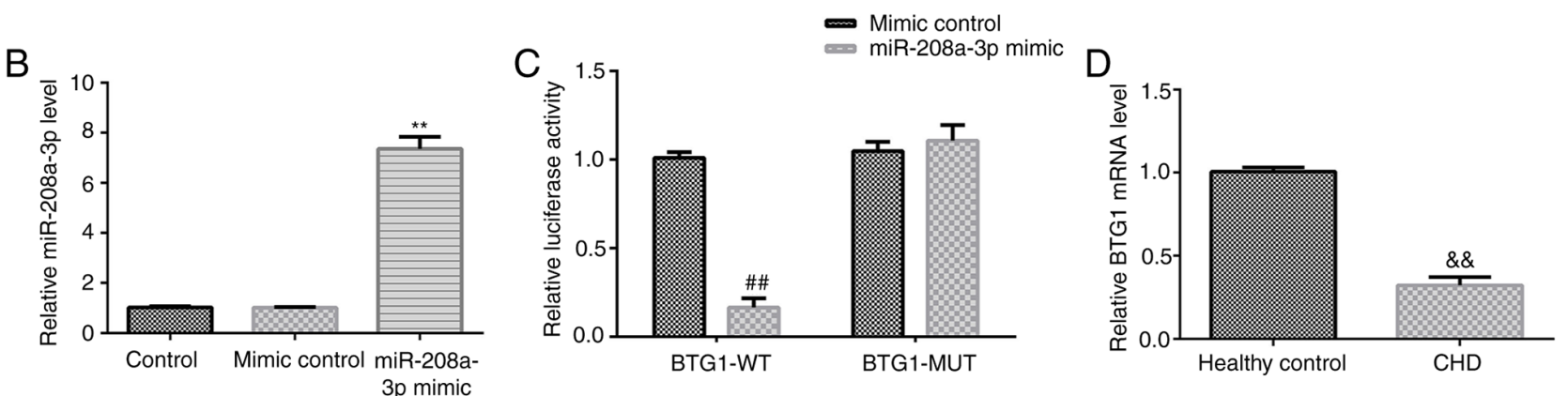

Figure 2. miR-208a-3p inhibits BTG1 expression by targeting the BTG1 transcript 3'-UTR. (A) Schematic representation of the 3'-UTR of BTG1 containing miR-205-5p binding sequences. (B) Expression of miR-208a-3p in human vascular smooth muscle cells transfected with mimic control or miR-208a-3p mimic. (C) Relative luciferase activities were determined by dual-luciferase assay at $48 \mathrm{~h}$ after transfection. (D) mRNA expression of BTG1 in the blood samples of patients with CHD and health individuals. ${ }^{* *} \mathrm{P}<0.01$ vs. control; ${ }^{\# \#} \mathrm{P}<0.01$ vs. mimic control; ${ }^{\& \&} \mathrm{P}<0.01$ vs. healthy control. BTG1, B-cell translocation gene 1; CHD, coronary heart disease; miR-208a-3p, microRNA-208a-3p; 3'-UTR, 3'-untranslated region.
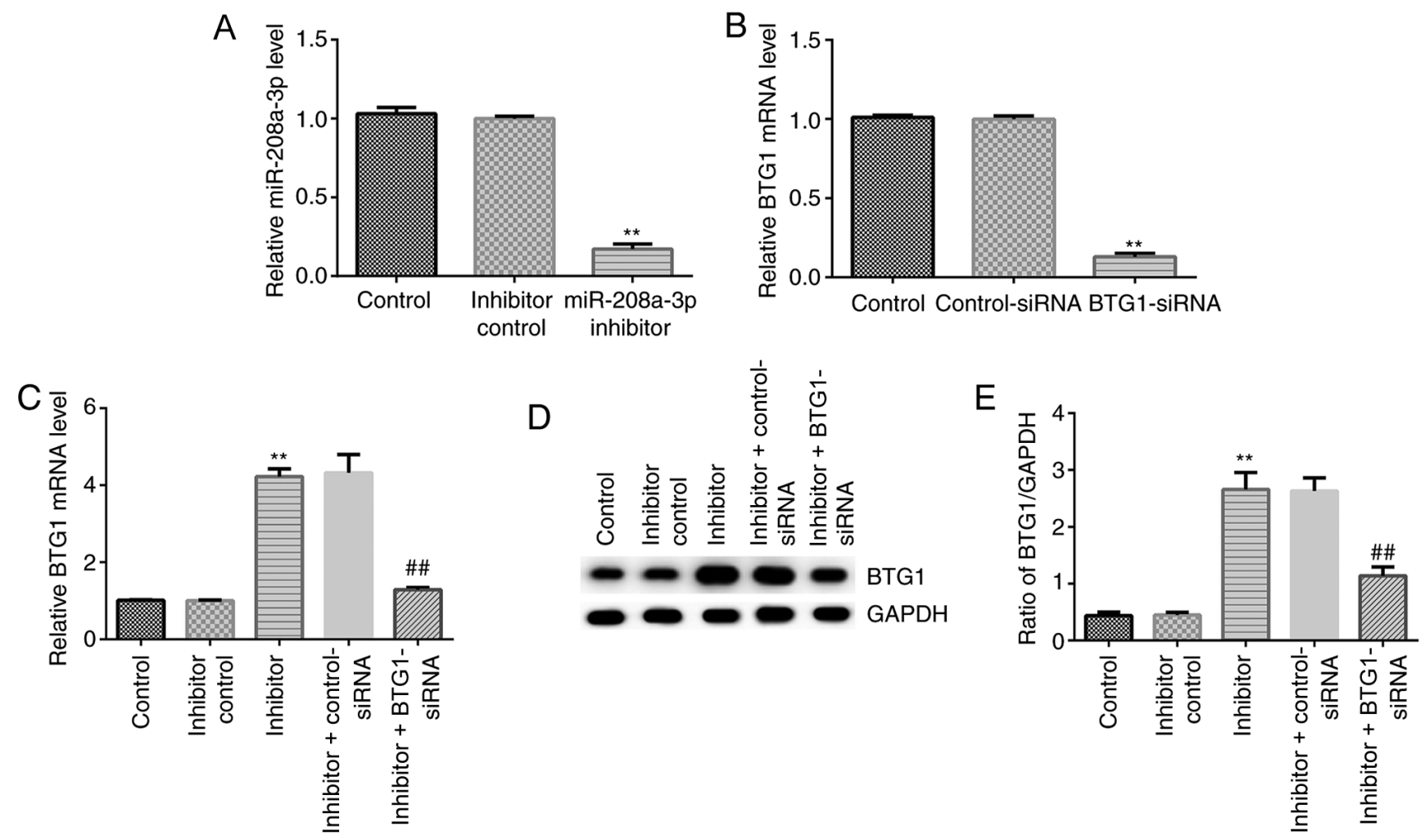

Figure 3. Effect of miR-208a-3p inhibitor on BTG1 expression in hVSMCs. (A) Detection of miR-208a-3p expression after hVSMCs were transfected with miR-208a-3p inhibitor or inhibitor control. (B) mRNA expression of BTG1 was determined in hVSMCs transfected with control-siRNA or BTG1-siRNA. (C) BTG1 mRNA expression in hVSMCs transfected with inhibitor control, miR-208a-3p inhibitor, miR-208a-3p inhibitor + control-siRNA or miR-208a-3p inhibitor + BTG1-siRNA. (D) Western blot analysis was performed to evaluate the BTG1 protein expression levels in hVSMCs transfected with inhibitor control, miR-208a-3p inhibitor, miR-208a-3p inhibitor + control-siRNA or miR-208a-3p inhibitor + BTG1-siRNA. (E) Ratio of BTG1/GAPDH was calculated and presented. ${ }^{* *} \mathrm{P}<0.01$ vs. control; ${ }^{\# \#} \mathrm{P}<0.01$ vs. inhibitor. BTG1, B-cell translocation gene 1; miR-208a-3p, microRNA-208a-3p; hVSMCs, human vascular smooth muscle cells; small interfering RNA.

demonstrated that miR-208a-3p inhibitor induced hVSMC cell apoptosis compared with the inhibitor control, while these effects were significantly attenuated by inhibiting BTG1 (Fig. 4B and C). Western blot analysis was also performed to investigate apoptosis-associated proteins, such as Bcl-2 and Bax. As shown in Fig. 4D-F, miR-208a-3p inhibitor significantly reduced Bcl-2 protein expression levels compared with the control, while it increased the expression level of Bax. However, BTG1-siRNA efficiently reversed the effects of miR-208a-3p inhibitor on Bax and $\mathrm{Bcl}-2$ protein expression in hVSMCs. These results suggested that miR-208a-3p was involved in hVSMC proliferation and apoptosis by targeting BTG1. 
A

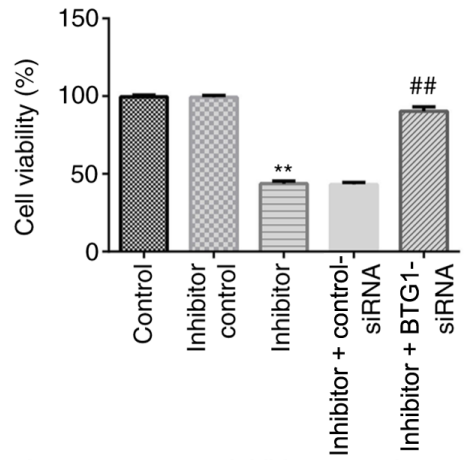

B
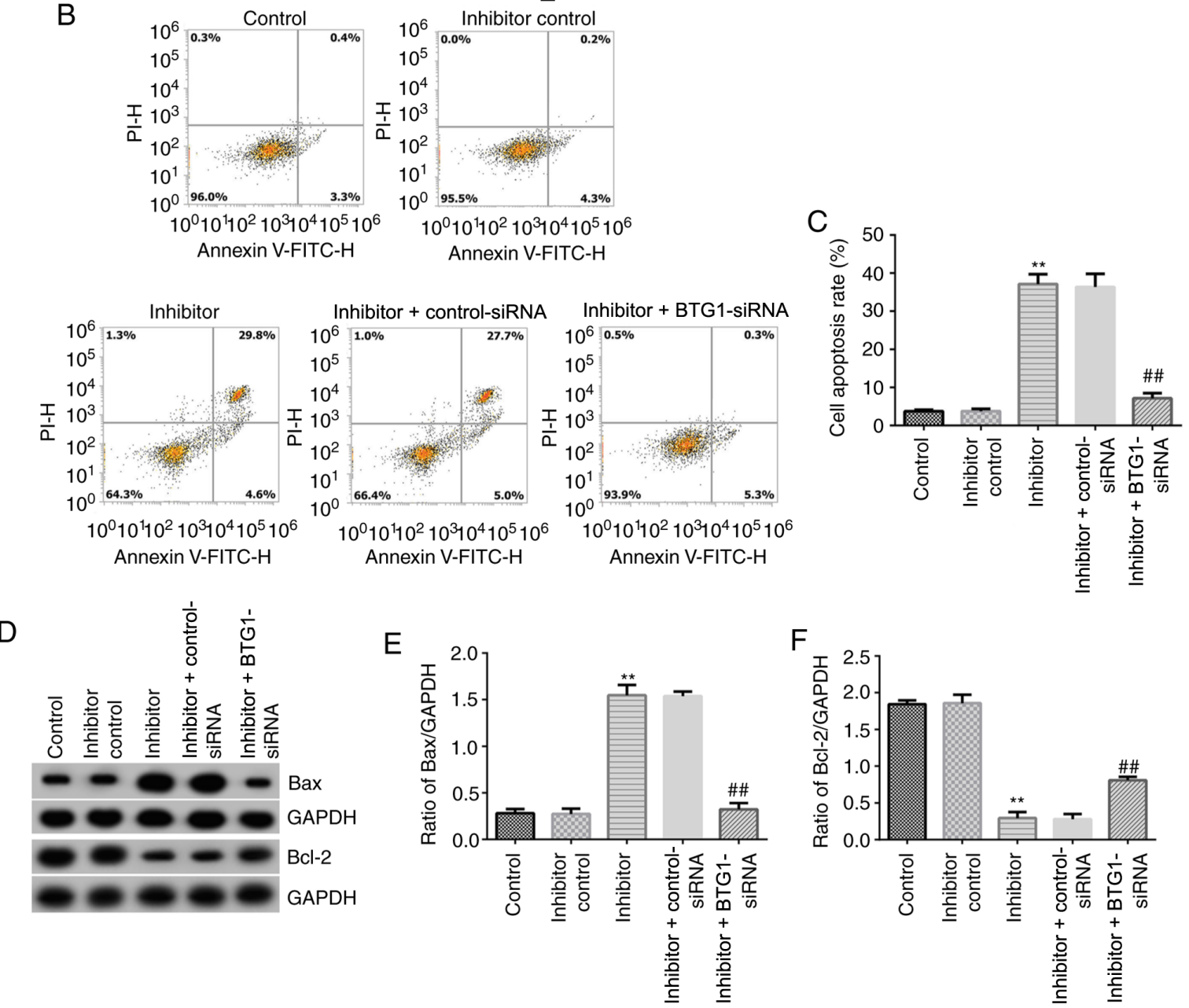

Figure 4. Effects of miR-208a-3p inhibitor or BTG1-siRNA on the viability and apoptosis of hVSMCs. (A) MTT assay was conducted to detect the viability of hVSMCs transfected with inhibitor control, miR-208a-3p inhibitor, miR-208a-3p inhibitor + control-siRNA or miR-208a-3p inhibitor + BTG1-siRNA for $48 \mathrm{~h}$. (B) Flow cytometry was performed $48 \mathrm{~h}$ after the transfection of hVSMCs with inhibitor control, miR-208a-3p inhibitor, miR-208a-3p inhibitor + control-siRNA or miR-208a-3p inhibitor + BTG1-siRNA. (C) Quantitative analysis of apoptotic cell ratio. (D) Protein expression levels of Bax and Bcl-2 were detected via western blot analysis in hVSMCs transfected with inhibitor control, miR-208a-3p inhibitor, miR-208a-3p inhibitor + control-siRNA or miR-208a-3p inhibitor + BTG1-siRNA for $48 \mathrm{~h}$. (E) Ratio of Bax/GAPDH was calculated and presented. (F) Ratio of Bcl-2/GAPDH was calculated and presented. ${ }^{* *} \mathrm{P}<0.01$ vs. control; ${ }^{\# \#} \mathrm{P}<0.01$ vs. inhibitor. BTG1, B-cell translocation gene 1; CHD, coronary heart disease; miR-208a-3p, microRNA-208a-3p; hVSMCs, human vascular smooth muscle cells; siRNA, small interfering RNA; PI, propidium iodide.

miR-208a-3p inhibitor affects the apoptosis of hVSMCs by regulating the PI3K/AKT pathway. The potential molecular mechanism of miR-208a-3p inhibitor was further investigated in terms of inducing cell apoptosis. Western blot analysis was conducted to detect relative protein expression levels, including p-AKT and AKT in hVSMCs (Fig. 5A). The ratio of p-AKT/AKT levels (Fig. 5B) was significantly suppressed in the miR-208a-3p inhibitor group compared with the control group; BTG1-siRNA significantly reversed these effects.
Effects of BTG1-siRNA on cell viability, apoptosis and the PI3K/AKT pathway in hVSMCs. To further explore the role of BTG1 in hVSMCs, the effect of BTG1-siRNA transfection alone on cell viability, apoptosis and PI3K/AKT activity were investigated in hVSMCs. It was observed that compared with the control group, BTG1-siRNA significantly promoted cell viability (Fig. 6A), inhibited cell apoptosis (Fig. 6B and C), and enhanced AKT phosphorylation (Fig. 6D and E) in hVSMCs. These findings further 

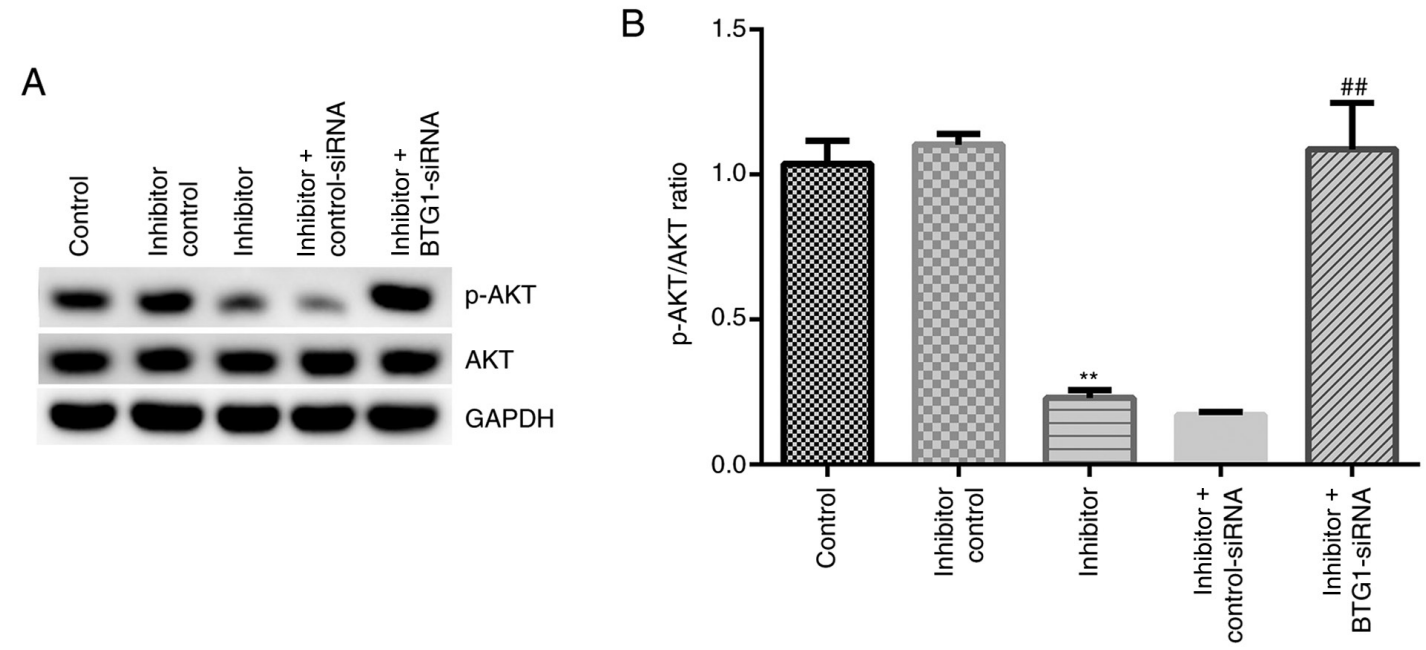

Figure 5. Effect of miR-208a-3p inhibitor on the PI3K/AKT pathway in hVSMCs. hVSMCs were transfected with inhibitor control, miR-208a-3p inhibitor, miR-208a-3p inhibitor + control-siRNA or miR-208a-3p inhibitor + BTG1-siRNA. (A) Detection of p-AKT and AKT protein levels in hVSMCs using western blot analysis. (B) Ratio of p-AKT/AKT was detected and presented. ${ }^{* *} \mathrm{P}<0.01$ vs. control; ${ }^{\# \#} \mathrm{P}<0.01$ vs. inhibitor. BTG1, B-cell translocation gene 1 ; miR-208a-3p, microRNA-208a-3p; hVSMCs, human vascular smooth muscle cells; siRNA, small interfering RNA; p, phosphorylated.
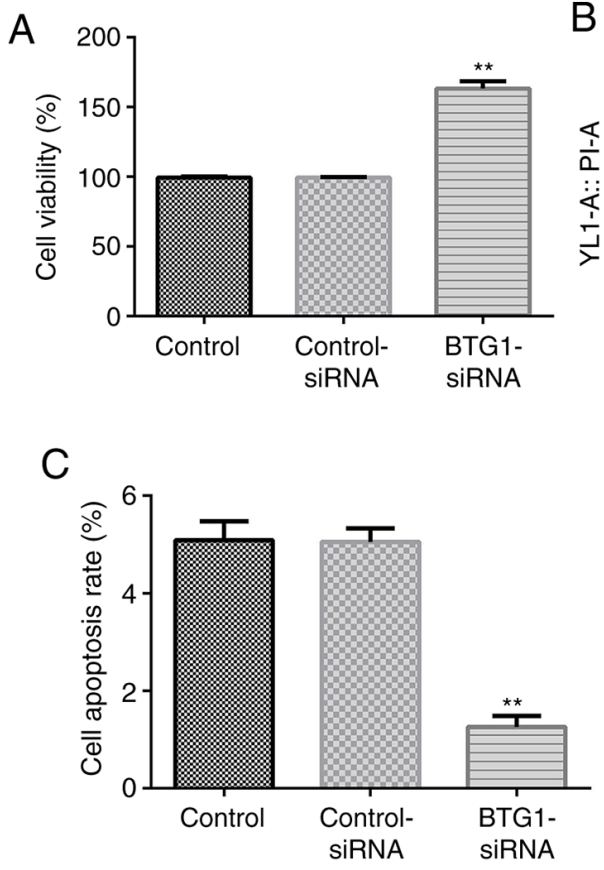
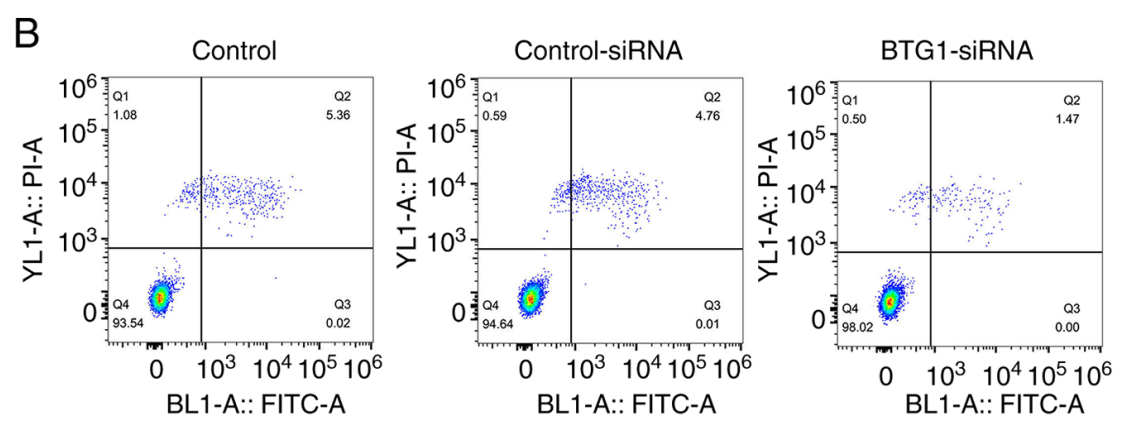

$\mathrm{D}$

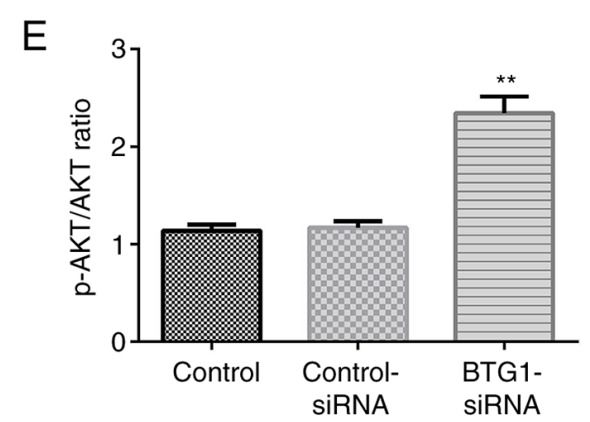

Figure 6. Effect of BTG1-siRNA on cell viability, apoptosis and the PI3K/AKT pathway in hVSMCs. hVSMCs were transfected with control-siRNA or BTG1-siRNA for $48 \mathrm{~h}$. (A) MTT assay was conducted to detect the viability of hVSMCs. (B) Flow cytometry was performed $48 \mathrm{~h}$ after transfection. (C) Quantitative analysis of apoptotic cell ratio. (D) Detection of p-AKT and AKT protein levels in hVSMCs using western blot analysis. (E) Ratio of p-AKT/AKT was detected and presented. "** $\mathrm{P}<0.01$ vs. control. BTG1, B-cell translocation gene 1; miR-208a-3p, microRNA-208a-3p; hVSMCs, human vascular smooth muscle cells; siRNA, small interfering RNA; PI, propidium iodide; p, phosphorylated.

suggested that BTG1 served a role in CHD development through effects on hVSMC function.

\section{Discussion}

It has been previously reported that abnormal regulation of hVSMCs may lead to the development of cardiovascular disease (21). Studies have demonstrated that miRNAs participate in the function of VSMCs, such as miRNA-21 (22) and miRNA-214 (23). Therefore, it is necessary to identify the specific miRNAs associated with the disease and their respective targets to further understand their role and examine novel therapeutic strategies for disease treatment. Previous reports have indicated that miR-208a-3p is involved in a number of diseases, including atrial fibrillation (24) and pathological cardiac hypertrophy (25). In addition, a previous study has suggested that miR-208a-3p is involved in acute cardiac injury (26). However, to the best of our knowledge, there are a limited number of reports investigating the expression levels and roles of miR-208a-3p in CHD. 
To understand the function of miR-208a-3p in CHD, the expression levels of miR-208a-3p were initially determined in the peripheral blood samples of 30 patients with $\mathrm{CHD}$ and healthy volunteers. The results showed that miR-208a-3p expression levels were significantly upregulated in patients with CHD compared with healthy individuals, suggesting that miR-208a-3p may serve important roles in CHD progression. This study also revealed that miR-208a-3p directly targeted the BTG1 3'-UTR by performing a dual-luciferase reporter assay. BTG1, which is a member of the pro-apoptotic Bcl-2 family, has been reported to be involved in multiple biological behaviors, including apoptosis and factor translocation regulation (27). Studies have shown that BTG1 plays an important role in regulating cell proliferation and apoptosis $(19,28)$, and it has been found to be a target gene of multiple miRNAs $(28,29)$. Furthermore, the present study found that BTG1 was downregulated in patients with $\mathrm{CHD}$. Thus, it was hypothesized that miR-208a-3p may participate in CHD by regulating hVSMC function via targeting of BTG1.

Then, the present study examined the functional mechanism through which miR-208a-3p regulated hVSMCs. The results demonstrated that miR-208a-3p inhibitor significantly inhibited cell viability and promoted apoptosis, whereas downregulation of BTG1 reversed these effects. Furthermore, miR-208a-3p participated in the regulation of apoptosis-related proteins, including Bcl-2 and Bax (30). These results suggested that miR-208a-3p may regulate VSMC proliferation and apoptosis in CHD. Therefore, the present study examined potential signaling pathways involved in miR-208a-3p-regulated apoptosis in hVSMCs, and the PI3K/AKT pathway (31) was analyzed in this study. PI3K/AKT signaling has also been reported to be involved in CHD development (32-34). For example, miR-26a-5p inhibition induced endothelial cell apoptosis by inhibiting the PI3K/AKT pathway (33). Decoy receptor-3 could regulate inflammation and apoptosis by regulating the $\mathrm{PI} 3 \mathrm{~K} / \mathrm{AKT}$ signaling pathway in a mouse model of coronary artery disease (33). The results of the present study indicated that miR-208a-3p inhibitor could inhibit the PI3K/AKT signaling pathway in hVSMCs, and in turn, this inhibition was abolished by BTG1-siRNA.

Finally, to further investigate the reversal effect of BTG1-siRNA on miR-208a-3p inhibitor-transfected hVSMCs, the effect of BTG1-siRNA on hVSMCs was determined. The data suggested that BTG1-siRNA could promote cell viability, inhibit cell apoptosis and promote the PI3K/AKT signaling pathway in hVSMCs. The data further suggested that miR-208a-3p may serve a role in CHD development by targeting BTG1.

In summary, these findings provided novel insight into the involvement of miR-208a-3p in the progression of CHD by regulating the proliferation of hVSMCs via downregulation of BTG1. This study provided a basis for understanding the functional mechanism of miR-208a-3p in CHD. However, this study is only a preliminary study of miR-208-3p in CHD. In order to verify the role of miR-208-3p in CHD, further in-depth studies are required. For example, the effect of BTG1-siRNA on hVSMCs requires further investigation. The role of miR-208-3p/BTG1 in CHD should also be investigated in vivo. The association between the expression of miR-208-3p/BTG1 and the clinicopathological features of patients with CHD also requires further exploration. In addition, examining other targets of miR-208a-3p in CHD therapy is critical.

\section{Acknowledgements}

Not applicable.

\section{Funding}

No funding was received.

\section{Availability of data and materials}

The datasets used and/or analyzed during the current study are available from the corresponding author on reasonable request.

\section{Authors' contributions}

DW contributed to data collection, statistical analysis, data interpretation and manuscript preparation. CY contributed to data collection and data interpretation. DW and CY confirm the authenticity of all the raw data. All authors have read and approved the final manuscript.

\section{Ethics approval and consent to participate}

Written informed consent was provided from all participants approving the use of their samples in the present study. All study protocols were approved by the Ethics Committee of Shanxi Bethune Hospital (Taiyuan, China).

\section{Patient consent for publication}

Not applicable.

\section{Competing interests}

The authors declare that they have no competing interests.

\section{References}

1. Hajek C, Guo X, Yao J, Hai Y, Johnson WC, Frazier-Wood AC, Post WS, Psaty BM, Taylor KD and Rotter JI: Coronary heart disease genetic risk score predicts cardiovascular disease risk in men, not women. Circ Genom Precis Med 11: e002324, 2018.

2. Olubowale OT, Safford MM, Brown TM, Durant RW, Howard VJ, Gamboa C, Glasser SP, Rhodes JD and Levitan EB: Comparison of expert adjudicated coronary heart disease and cardiovascular disease mortality with the national death index: Results from the REasons for geographic and racial differences in stroke (REGARDS) study. J Am Heart Assoc 6: e004966, 2017.

3. Yoshioka S, Tsukamoto $\mathrm{T}$ and Chihara K: Vascular smooth muscle cells in coronary heart disease. Nihon Rinsho 61 (Suppl 4): S80-S85, 2003 (In Japanese).

4. Wang S, Cheng Z and Chen X: Promotion of PTEN on apoptosis through PI3K/Akt signal in vascular smooth muscle cells of mice model of coronary heart disease. J Cell Biochem 120: 14636-14644, 2019.

5. Lacolley P, Regnault V, Segers P and Laurent S: Vascular smooth muscle cells and arterial stiffening: Relevance in development, aging, and disease. Physiol Rev 97: 1555-1617, 2017.

6. Maier KG, Ruhle B, Stein JJ, Gentile KL, Middleton FA and Gahtan V: Thrombospondin-1 differentially regulates microRNAs in vascular smooth muscle cells. Mol Cell Biochem 412: 111-117, 2016. 
7. Cheuk BL and Cheng SW: Identification and characterization of microRNAs in vascular smooth muscle cells from patients with abdominal aortic aneurysms. J Vasc Surg 59: 202-209, 2014.

8. Matuszcak C, Lindner K, Eichelmann AK, Hussey DJ, Haier J and Hummel R: MicroRNAs: Key regulators of chemotherapy response and metastatic potential via complex control of target pathways in esophageal adenocarcinoma. Surg Oncol 27 392-401, 2018.

9. Pio G, Ceci M, D'Elia D, Loglisci C and Malerba D: A novel biclustering algorithm for the discovery of meaningful biological correlations between microRNAs and their target genes. BMC Bioinformatics 14 (Suppl 7): S8, 2013.

10. De Rosa S and Indolfi C: Circulating microRNAs as biomarkers in cardiovascular diseases. Exp Suppl 106: 139-149, 2015.

11. Fernandes T, Soci UP and Oliveira EM: Eccentric and concentric cardiac hypertrophy induced by exercise training: MicroRNAs and molecular determinants. Braz J Med Biol Res 44: 836-847, 2011.

12. Lin RC, Weeks KL, Gao XM, Williams RB, Bernardo BC, Kiriazis H, Matthews VB, Woodcock EA, Bouwman RD, Mollica JP, et al: PI3K(p110 alpha) protects against myocardial infarction-induced heart failure: Identification of PI3K-regulated miRNA and mRNA. Arterioscler Thromb Vasc Biol 30: 724-732, 2010.

13. Wang H, Lu J, Wu S, Yang S, Wang L, Zhou H, Fu Y and Liu J: Effects of electroacupuncture at different acupoints on apoptosis and the expression of miRNAs in myocardial cells in rats model of myocardial ischemia. Zhongguo Zhen Jiu 36: 281-286, 2016 (In Chinese).

14. Seo HH, Lee SY, Lee CY, Kim R, Kim P, Oh S, Lee H, Lee MY, Kim J, Kim LK, et al: Exogenous miRNA-146a enhances the therapeutic efficacy of human mesenchymal stem cells by increasing vascular endothelial growth factor secretion in the ischemia/reperfusion-injured heart. J Vasc Res 54: 100-108, 2017.

15. Wu H, Xu L, Chen Y and Xu C: MiR-208a-3p functions as an oncogene in colorectal cancer by targeting PDCD4. Biosci Rep 39: BSR20181598, 2019.

16. Montgomery RL, Hullinger TG, Semus HM, Dickinson BA, Seto AG, Lynch JM, Stack C, Latimer PA, Olson EN and van RooijE: Therapeutic inhibition of miR-208a improves cardiac function and survival during heart failure. Circulation 124: 1537-1547, 2011

17. Cui HB, Ge HE, Wang YS and Bai XY: MiR-208a enhances cell proliferation and invasion of gastric cancer by targeting SFRP1 and negatively regulating MEG3. Int J Biochem Cell Biol 102: 31-39, 2018

18. Hortmann M, Walter JE, Benning L, Follo M, Mayr RM, Honegger U, Robinson S, Stallmann D, Duerschmied D, Twerenbold R, et al: Droplet digital PCR of serum miR-499, miR-21 and miR-208a for the detection of functionally relevant coronary artery disease. Int J Cardiol 275: 129-135, 2019.

19. Zhao S, Chen SR, Yang XF, Shen DF, Takano Y, Su RJ and Zheng HC: BTG1 might be employed as a biomarker for carcinogenesis and a target for gene therapy in colorectal cancers. Oncotarget 8: 7502-7520, 2017.

20. Livak KJ and Schmittgen TD: Analysis of relative gene expression data using real-time quantitative PCR and the 2(-Delta Delta C(T)) method. Methods 25: 402-408, 2001.

21. Wu ZW, Liu YF, Wang S and Li B: MiRNA-146a induces vascular smooth muscle cell apoptosis in a rat model of coronary heart disease via NF-kappaB pathway. Genet Mol Res 14: $18703-18712,2015$.
22. Li FP, Lin DQ and Gao LY: LncRNA TUG1 promotes proliferation of vascular smooth muscle cell and atherosclerosis through regulating miRNA-21/PTEN axis. Eur Rev Med Pharmacol Sci 22: 7439-7447, 2018

23. Afzal TA, Luong LA, Chen D, Zhang C, Yang F, Chen Q, An W, Wilkes E, Yashiro K, Cutillas PR, et al: NCK associated protein 1 modulated by miRNA-214 determines vascular smooth muscle cell migration, proliferation, and neointima hyperplasia. J Am Heart Assoc 5: e004629, 2016.

24. Li S, Jiang Z, Wen L, Feng G and Zhong G: MicroRNA-208a-3p contributes to connexin40 remolding in human chronic atrial fibrillation. Exp Ther Med 14: 5355-5362, 2017.

25. Wang L, Ye N, Lian X, Peng F, Zhang $\mathrm{H}$ and Gong $\mathrm{H}$ : MiR-208a-3p aggravates autophagy through the PDCD4-ATG5 pathway in Ang II-induced $\mathrm{H} 9 \mathrm{c} 2$ cardiomyoblasts. Biomed Pharmacother 98: 1-8, 2018

26. Glineur SF, De Ron P, Hanon E, Valentin JP, Dremier S and Nogueira da Costa A: Paving the route to plasma miR-208a-3p as an acute cardiac injury biomarker: Preclinical rat data supports its use in drug safety assessment. Toxicol Sci 149: 89-97, 2016.

27. Huang Y, Zheng J, Tan T, Song L, Huang S, Zhang Y, Lin L, Liu J, Zheng P, Chen X, et al: BTG1 low expression in pancreatic ductal adenocarcinoma is associated with a poorer prognosis. Int J Biol Markers 33: 189-194, 2018.

28. Su C, Huang DP, Liu JW, Liu WY and Cao YO: MiR-27a-3p regulates proliferation and apoptosis of colon cancer cells by potentially targeting BTG1. Oncol Lett 18: 2825-2834, 2019.

29. Zhao X, Chen GQ and Cao GM: Abnormal expression and mechanism of miR-330-3p/BTG1 axis in hepatocellular carcinoma. Eur Rev Med Pharmacol Sci 23: 6888-6898, 2019.

30. Hassan M, Watari H, AbuAlmaaty A, Ohba Y and Sakuragi N: Apoptosis and molecular targeting therapy in cancer. Biomed Res Int 2014: 150845, 2014.

31. Reddy D, Kumavath R, Tan TZ, Ampasala DR and Kumar AP: Peruvoside targets apoptosis and autophagy through MAPK Wnt $/ \beta$-catenin and $\mathrm{PI} 3 \mathrm{~K} / \mathrm{AKT} / \mathrm{mTOR}$ signaling pathways in human cancers. Life Sci 241: 117147, 2020.

32. Feng H, Wang Z, Wang C, Zhu X, Liu Z, Liu H, Guo M, Hou Q and $\mathrm{Chu} Z$ : Effect of furostanol saponins from allium macrostemon bunge bulbs on platelet aggregation rate and pi3k/akt pathway in the rat model of coronary heart disease. Evid Based Complement Alternat Med 2019: 9107847, 2019.

33. Jing R, Zhong QQ, Long TY, Pan W and Qian ZX: Downregulated miRNA-26a-5p induces the apoptosis of endothelial cells in coronary heart disease by inhibiting PI3K/AKT pathway. Eur Rev Med Pharmacol Sci 23: 4940-4947, 2019.

34. Chen X, Wang R, Chen W, Lai L and Li Z: Decoy receptor-3 regulates inflammation and apoptosis via PI3K/AKT signaling pathway in coronary heart disease. Exp Ther Med 17: 2614-2622, 2019.

This work is licensed under a Creative Commons Attribution-NonCommercial-NoDerivatives 4.0 International (CC BY-NC-ND 4.0) License. 